\title{
Realistic simulation of BM@N GEM detectors: model implementation and testing
}

\author{
Alexander Zinchenko1', Tatiana Mamontova², Mikhail Kapishin \\ 1Joint Institute for Nuclear Research, Dubna, Russia \\ ${ }^{2}$ National Research Nuclear University MEPhl, Moscow, Russia
}

\section{Abstract}

The Lorentz shift of electrons in a magnetic field is taken into account with simulating the response of a GEM detector for the BM@N experiment. Dependences of the determined coordinate $x$ on the track angle, the values of the Lorentz shift of electrons and its dispersion for the gas mixture $\mathrm{ArC}_{4} \mathrm{H}_{10}$ were obtained. The momentum resolution was obtained as a function of the momentum for the deuteron-carbon $(\mathrm{dC})$ process, and the invariant mass of the $\Lambda$-hyperon was determined. The obtained

Corresponding Author: Alexander Zinchenko mamontova.tat.v@gmail.com

Received: 25 December 2017

Accepted: 2 February 2018

Published: 9 April 2018

Publishing services provided by Knowledge E

(c) Alexander Zinchenko et al. This article is distributed under the terms of the Creative Commons Attribution License, which permits unrestricted use and redistribution provided that the original author and source are credited.

Selection and Peer-review under the responsibility of the ICPPA Conference Committee. results are close to the first results of experimental data collected in the technical run in interactions of the deuteron beam of $4 \mathrm{AGeV}$ with different targets.

\section{Introduction}

Relativistic heavy ion collisions provide the unique opportunity to study nuclear matter in a wide scope from moderate to extreme densities and temperatures. In the collisions at moder- ate temperatures nucleons are excited to baryonic resonances which decay by the emission of mesons. The ratio of produced mesons to baryons increases with the collision energy. Heavy-ion collisions are a rich source of strangeness, and the coalescence of lambda-hyperons with nucleons can produce a variety of light hypernuclei. The study of the hyper-nuclei production is expected to provide new insights into the properties of the hyperon-nucleon and hyperon- hyperon interactions. [1]-[2]

BM@N experiment (Barionic Matter at Nuclotron) is located in Join Institute of Nuclear Research and is included in the future complex NICA (Nuclotron-based Ion Collider fAcility). The aim of BM@N experiment is to study the interaction of relativistic heavy ion beams with fixed targets.

\section{G OPEN ACCESS}




\section{Detector geometry}

The schematic view of the proposed experimental setup is shown in Fig. 1. The detailed description of the BM@N geometry can be found in Ref. [3]. The experiment combines measurement of track parameters with high accuracy with time-of-flight information for the particle identification and involves measurement of the total energy for analyzing the centrality of collisions.

As a typical fixed-target spectrometer, BM@N contains a dipole analyzing magnet. The gap between the poles of the magnet is around $1 \mathrm{~m}$. The magnetic field can be varied up to $1.2 \mathrm{~T}$ to get the optimal BM@N detector acceptance and momentum resolution for different processes and beam energies.

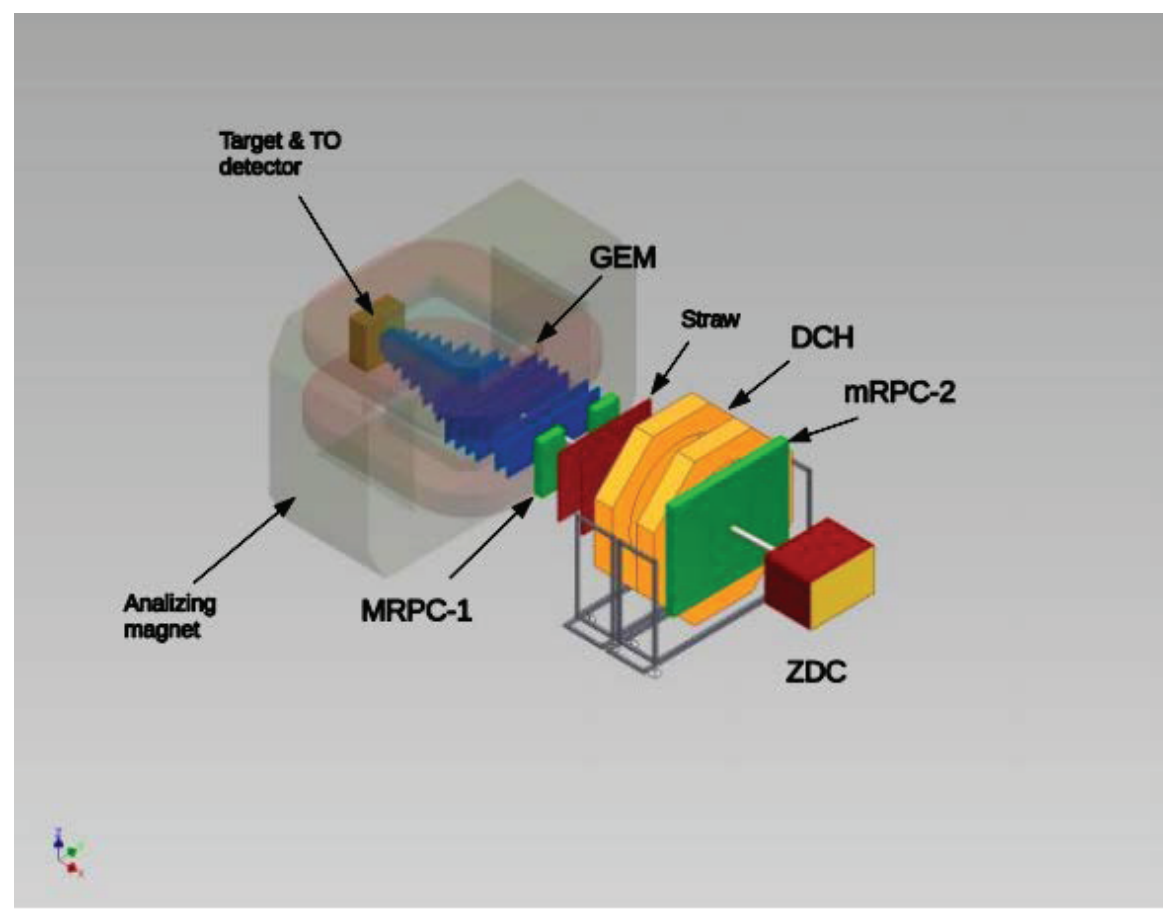

Figure 1: The schematic view of the BM@N experiment.

The central track system of the BM@N experiment is based on 2-coordinate Detectors GEM (Gas Electron Multiplier), each detector with 3 electrodes- multipliers of electrons. The GEM detectors are manufactured using technology, developed at CERN. They have already been used in various experiments (COMPASS, JLAB, STAR, CMS). The signal recording is done from the anode plane using the doube-sided strip readout technique with a strip pitch of $800 \mu \mathrm{m}$ and strip orientation of o and $15^{\circ}$ with respect to the vertical axis. The readout board is divided into two zones: the outer and inner ones. The inner (hot) zone with shorter strips will be located near the beam line and is intended to cope with a high particle flux in this region. 


\section{Simulation of BM@N GEM detectors}

\subsection{Description of the model}

At this moment active work is underway to simulate the BM@N experiment by the Monte Carlo method. Modeling for optimizing BM@N detectors is performed using the generated $\mathrm{Au}+\mathrm{Au}$ events. For this purpose, a special software environment BMNRoot was developed [4]. In order to obtain a realistic response of the facility to interactions, a detailed geometric description of each of the detectors was realized.

Our task was to take into account the Lorentz shift of drifting electrons in the modeling of the response of the GEM detector. The electrons formed after the passage of the particle through the GEM detector will shift relative to their initial position along the axis perpendicular to the plane of the electric and magnetic field vectors. The software GARFIELD ++ was used to determine the mean shifts of electrons under the action of Lorentz force. The structure of the GEM detector used in the simulation is presented in Fig. 2.

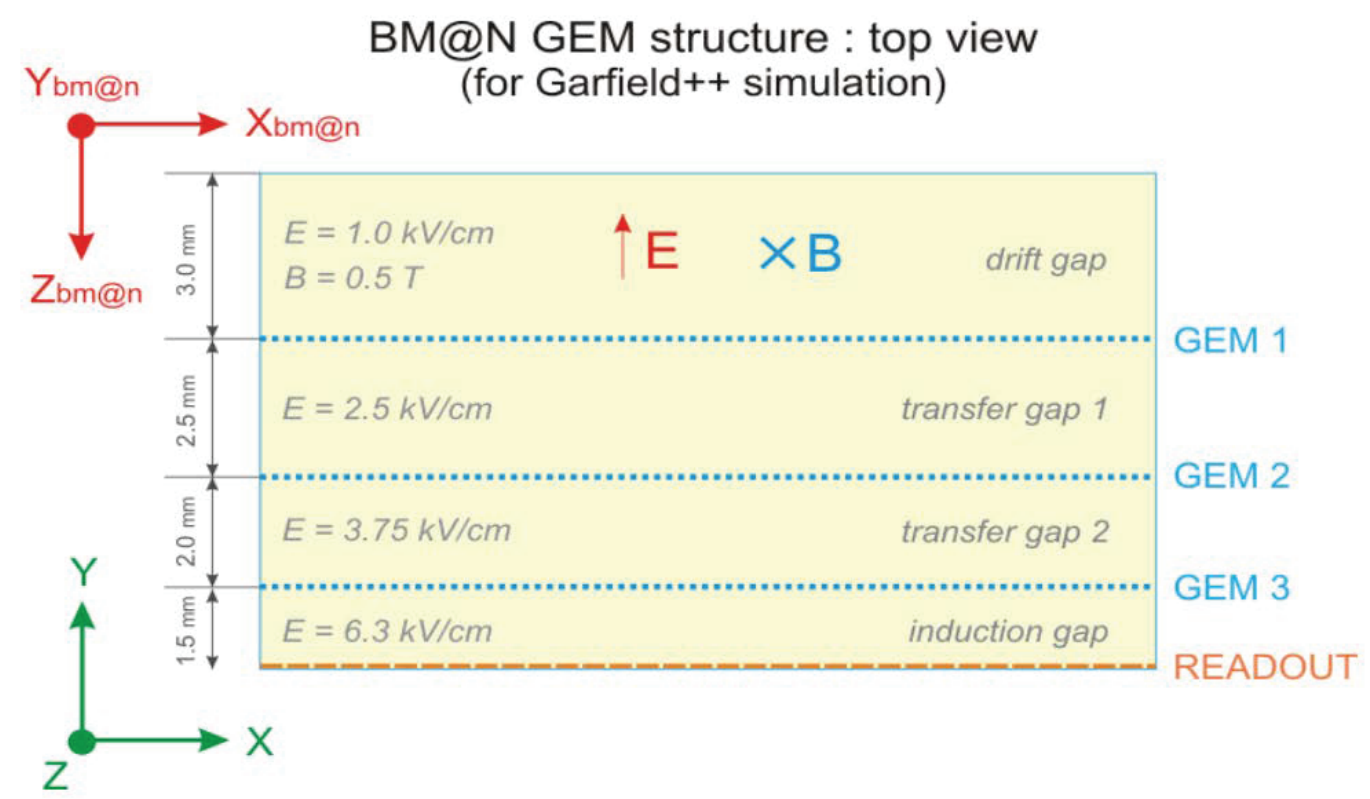

Figure 2: Structure and characteristics of $G E M$ module for $\operatorname{ArCO}_{2}(70 / 30)$ and fields: $E=$ 1000:2500:3750:6300 V/cm, B = $0.5 \mathrm{~T}$

The mean electron shift and their diffusion depend on the distance to the readout plane. These dependences were approximated with polynomials of different degrees. 
For the gas mixture $\mathrm{ArC}_{4} \mathrm{H}_{10}(90 / 10)$ and the fields $E=880: 1920: 2780: 3160$ $\mathrm{V} / \mathrm{cm}$ : the mean shift is approximated by a polynomial of the third degree with the following coefficients:

$$
p_{0}=0.000824255 ; p_{1}=0.156657 ; p_{2}=-0.17709 ; p_{3}=0.475568
$$

Diffusion is given by a polynomial of the fifth degree with the following coefficients:

$p_{0}=0.000252085 ; p_{1}=0.15543 ; p_{2}=-0.518142 ; p_{3}=1.03222 ; p_{4}=-0.97707 ; p_{5}=0.355$.

([5])

Also it is proposed that the electron drift has opposite directions for even and odd GEM stations.

\subsection{Results}

The results on the GEM coordinate resolution were obtained from the runs without the target in the magnetic field of 0.79 T. For the measure of the spatial accuracy the standard deviation of the gaussian fit (sigma) of the hit residuals was taken, where the hit residuals are the distributions of hit deviations from the reconstructed tracks. One can see that the obtained result of $\sim 0.67 \mathrm{~mm}$ is reproduced by the simulation. (Fig. 3)
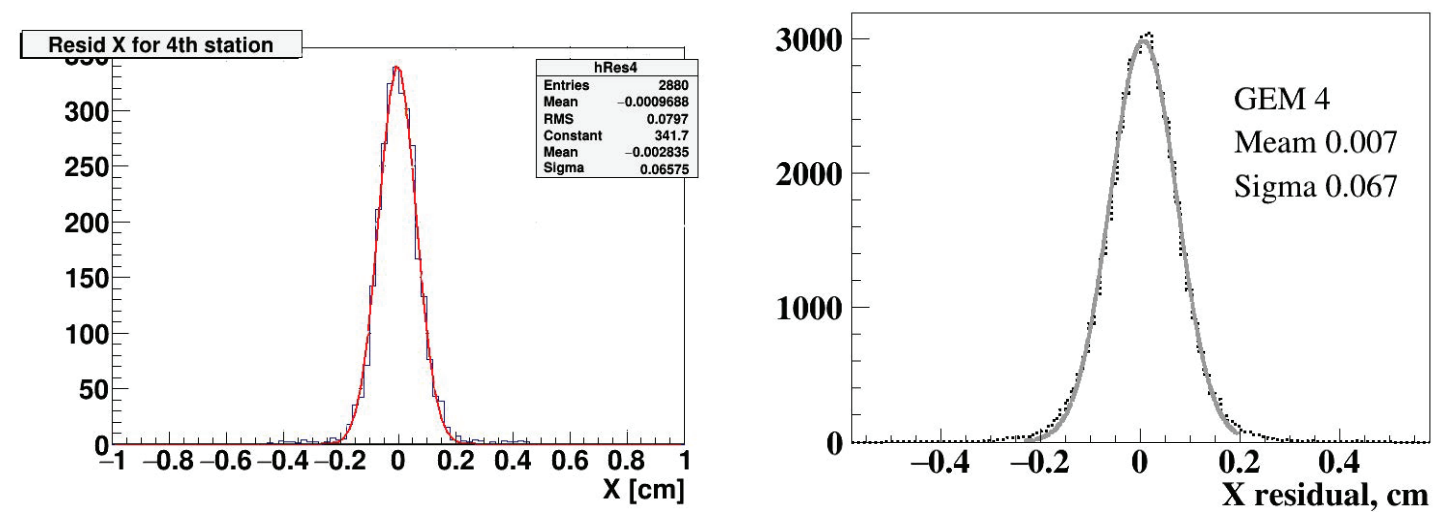

Figure 3: Comparison a residual $x$ for the 4 th station from the model (left) (tracks) and from the experiment (right).

The reconstructed track momentum distribution for the deuteron beam with a momentum of $9.7 \mathrm{GeV} / \mathrm{c}$ is shown on 4. The plot shows the relative momentum resolution as a function of momentum as obtained from simulation in comparison with the experiment. One can see a deviaton of the beam deuteron point from the linear dependence at lower momenta. This can be attributed to the difference in the angular range of tracks from different momentum domains, i.e. high momenta tracks 


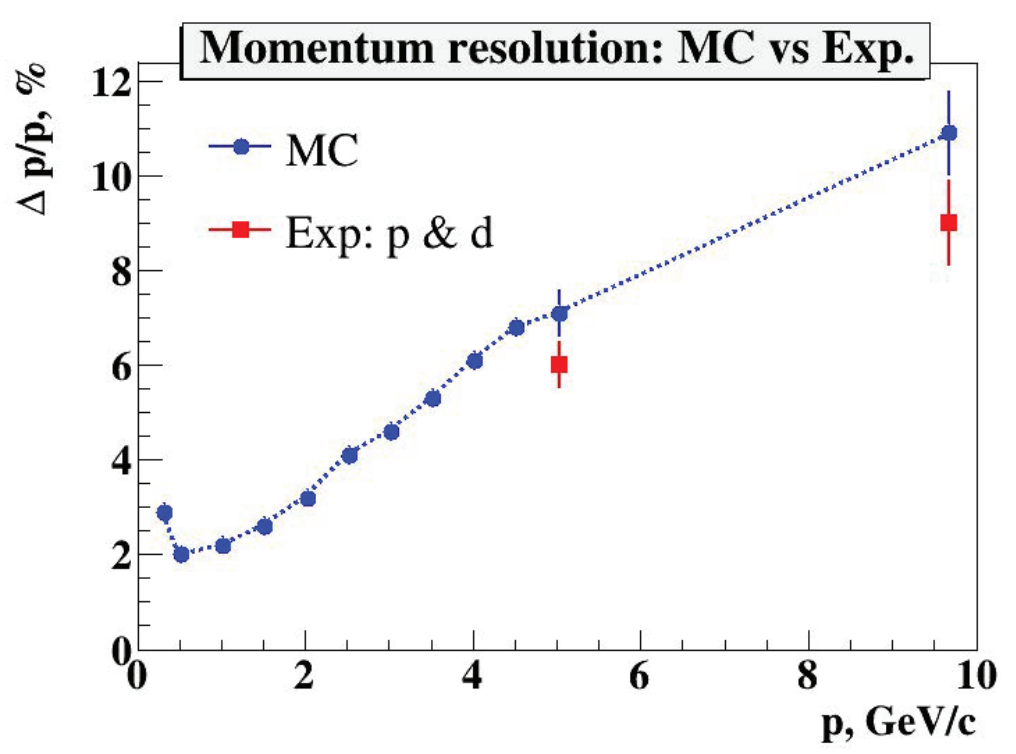

Figure 4: Momentum resolution dependence on the momentum.
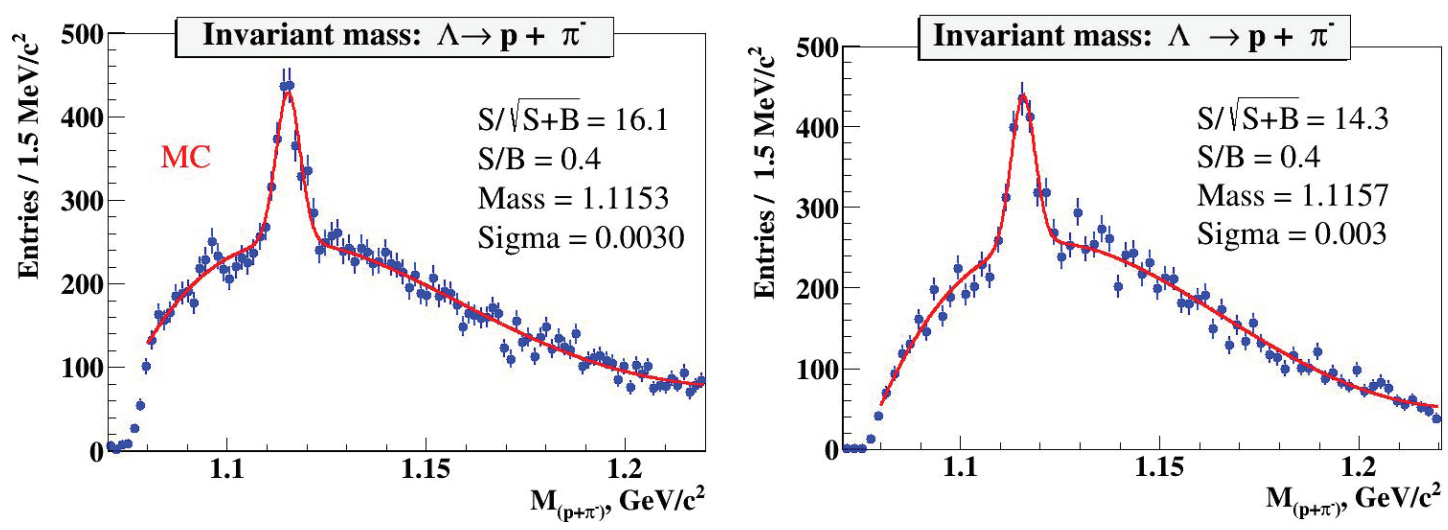

Figure 5: Invariant mass of $\Lambda$ from the model (left) and from the experiment (right).

went in almost normal direction to the detector planes. Therefore, the effect of the Lorentz angle was less pronounced as opposed to more inclined tracks.

Finally, we obtain the invariant mass of $\Lambda$-hyperon without the combinatorial background. Its value from the model equals to $1.116 \mathrm{GeV} / \mathrm{c}^{2}$ and $\sigma=0.0022 \mathrm{GeV} / \mathrm{c}^{2}$. These values are close to the experimental (Fig. 5 (right)).

\section{Acknowledgements}

This work was supported by the University Center of JINR and Veksler and Baldin Laboratory of High Energy Physics. Also this work was partially supported by the Ministry of Science and Education of the Russian Federation, grant N 3.3380.2017/4.6, and by the National Research Nuclear University MEPhl in the framework of the Russian Academic Excellence Project (contract No. 02.a03.21.0005, 27.08.2013). 


\section{References}

[1] J. Adams et al, Nucl. Phys. A 757, 102-183 (2005)

[2] K. Adcox et al, Nucl. Phys. A 757, 184-283 (2005)

[3] Studies of baryonic matter at the Nuclotron (BM@N) http://bmnshift.jinr.ru

[4] Simulation and Analysis Frameworks for MPD and BM@N at NICA http://mpd.jinr.ru

[5] Analysis meeting http://bmnshift.jinr.ru/wiki/doku.php?id=analysis_meetings 